\title{
Phase diagram of the polymeric nitrogen
}

\author{
L.N. Yakub \\ Thermophysics Department, Odessa National Academy of Food Technologies \\ 112 Kanatnaya Str., Odessa 65039, Ukraine \\ E-mail: unive@icn.od.ua
}

Received February 24, 2015, published online April 23, 2015

\begin{abstract}
The results of theoretical prediction of the phase transition lines of molecular nitrogen into the polymeric phase at high pressure are presented. The role of the polymeric phase structure in the location of the transition lines on the phase diagram is considered. Possible configuration of the melting curve of the polymeric nitrogen solid forming the polymeric liquid is discussed. Predicted volumes of the coexisting phases are compared with experimental data and with results of the computer simulations.

PACS: 64.70.dj Melting of specific substances;

64.70.D- Solid-liquid transitions;

61.43.Bn Structural modeling: serial-addition models, computer simulation.
\end{abstract}

Keywords: molecular solids, polymeric solid, polymeric liquid, melting, equation of state, phase equilibria.

\section{Introduction}

During last decades condensed systems consisting of simple molecules with multiple bonds, which under strong compression are able to form multiple coordinated atomic structures with the redistribution of chemical bonds, were intensively investigated [1]. Such substances allow at extreme conditions accumulating many times larger amount of energy than the high-energy-density materials used today. An excellent example of such type of substances is molecular nitrogen.

The first experimental evidence of the specific behavior of strongly compressed nitrogen was the effect of the "shock cooling" discovered on the Hugoniot curve of liquid nitrogen at high temperatures [2], which was interpreted as a molecular dissociation with the first-order transition in shock-compressed nitrogen fluid. Shock-wave experiments indicated a first-order phase transition with the volume jump of $1.5-2.0 \mathrm{~cm}^{3} / \mathrm{mol}$.

Theoretical studies [3-5] have suggested that this transition may be associated with the dissociation of nitrogen molecules into atoms (with breaking of the strong triple chemical bond), accompanied by formation of a set of ordinary chemical bonds between nearest neighbors. Such transition into an atomic (or polymeric [5]) phase was supposed to be possible in both the liquid and solid state. The first theoretical estimations [3,4] predicted that one of the most stable at $T=0 \mathrm{~K}$ atomic crystalline lattices should have a layered A7 (arsenic-like) structure. Such hypothetical transi- tion into the stable polymeric nonmetallic structure was expected to occur at a pressure of about $77 \mathrm{GPa}$ and have a significant (about 35\%) decrease in the volume [3].

Autors [5] reported a new $a b$ initio calculations, which predicted the spatial cubic gauche (CG) lattice, in which all atoms are triply coordinated, to be the most stable polymeric structure and to have the total energy slightly lower than the layered A7 structure. However, this theoretically predicted transition of molecular $\mathrm{N}_{2}$ low-temperature solid into the polymeric CG crystalline form was not observed for years what was attributed to a large energy barrier separating phases.

This theoretically predicted dissociation of the nitrogen molecules at high pressure in solid state has been confirmed experimentally relatively recently [6-9]. Only in 2004 Eremets and co-workers succeeded in synthesizing the crystalline form of triply coordinated polymeric nitrogen directly from the molecular nitrogen [9]. Diatomic nitrogen was first heated up to temperature above $2000 \mathrm{~K}$, compressed in the diamond anvils by pressure higher than $110 \mathrm{GPa}$, and then analyzed by x-ray diffraction and Raman spectroscopy. A limited number of x-ray diffraction peaks produced by the powder formed not allowed until recently confirming the existence of CG structure, but the x-ray diffraction on the single crystal obtained in Ref. 10 confirmed it. Thus, thanks to the long-lasting efforts of experimenters the existence of the transition of highly compressed molecular nitrogen into the nonmolecular (polymeric) phase has been convincingly proved. 
In the solid molecular nitrogen at least two highpressure phases, which can be directly transformed into the polymeric phase are known [11]. One of them ( $\zeta$-phase), may be in equilibrium with the polymeric solid phase of nitrogen at low temperatures, the second one ( $\varepsilon$-phase) at high temperatures, in the vicinity of the melting line.

These two phases differ from each other in the lattice type and in the character of molecular rotation. In the monoclinic $\zeta$-phase rotational motion is suppressed (only molecular librations are possible) and in the rhombohedral $\varepsilon$-phase molecular rotation is relatively free. The type of the crystalline structure of these phases has not been clearly established for a long time, although the region of stability of each was known [11].

In this regard a few $a b$ initio calculations of energy and relative stability performed for a number of crystal structures of the polymeric nitrogen in recent years [12,13] should be noted. They predicted the relative stability of the CG structure in the pressure range of 50 up to $150 \mathrm{GPa}$. However, the estimates of the CG stability region cannot be considered as completely reliable because they do not agree with the experimentally studied in this pressure range part of the phase diagram $[7,8]$ and were performed at $T=0 \mathrm{~K}$ relative to the high-temperature molecular $\varepsilon$-phase instead of the low-temperature $\zeta$-phase.

Thus, the configuration of the phase diagram of the nitrogen at elevated temperatures in the range of extremely high pressure up to the present time remains unclear. This gap has been partially filled by a number of theoretical works [14-18], and computer simulations [19,20].

The aim of this work is to discuss the results of these theoretical predictions concerning the region of coexistence between molecular and polymeric solid and liquid phases of nitrogen in a wide range of temperatures and extremely high pressures and consider the role of the polymeric phase structure in the shape of the phase diagram of strongly compressed nitrogen.

\section{Equations of state for high-pressure phases of solid nitrogen}

The theoretical prediction of location of the transition lines in the phase diagram is a task requiring usage of the equations of state. In the case of transitions without the change of symmetry (e.g., liquid-liquid transition), it is possible to apply a single (common) equation of state for both coexisting phases. For example, in [14] a single equation of state was developed on the basis of the modified "soft dumbbells-sticky spheres" model and was applied to the highly compressed nitrogen fluid.

Within this model the partially polymerized fluid was represented as a mixture of diatomic molecules $\mathrm{N}_{2}$, which were modeled by soft dumbbells (whose diameters and lengths of bonds depend on the temperature), with a set of different polymeric clusters modeled by sticky spheres capable of forming up to three rigid bonds to each other.
However, such approach is not applicable to the prediction of transitions between crystalline phases. Two different expressions for the free energy of coexisting phases (called canonical equations of state) are necessary here. Prediction of the pressure dependence of the equilibrium phase transition temperature $T(P)$ of the molecular nitrogen into the polymeric phase in [16-18] was made on the basis of two separate canonical equations of state, one for the polymeric, and other for the molecular high-pressure phase.

Due to small energy difference between two highpressure crystalline phases of molecular nitrogen mentioned above, in a number of studies (particularly in $[5,16]$ ) it was used an approximate $\left(\beta-\mathrm{O}_{2}\right.$-like) representation of the molecular structure of the high-pressure phase, and both these phases were described by a single equation of state.

This equation of state for molecular high-pressure phase of nitrogen was based on the atom-atom force crystal model [16], which takes into account both the repulsion of nonbonded atoms and "chemical attraction" of atoms joined by the triple bond in the $\mathrm{N}_{2}$ molecule. Parameters of this force model were calibrated on the results of $a b$ initio calculation of the molecular crystal energy [5].

Helmholtz free energy was presented in the form

$$
F_{\text {mol }}(T, V)=F_{\text {lat }}^{(\mathrm{id})}(T, V)+U_{\text {mol }}\left(R_{0}\right)-N_{m} k T \ln \left(\frac{v_{f}^{\mathrm{mol}}}{v}\right) \text {. }
$$

Here $F_{\text {lat }}^{(\mathrm{id})}(T, V)$ is the energy of an ideal molecular lattice gas as a function of temperature and volume, $U_{\text {mol }}\left(R_{0}\right)$ is the energy of static lattice, $R_{0}$ is the length of the (triple) chemical bond, $v=V / N_{m}$ and $v_{f}^{\mathrm{mol}}$ are the specific and so-called molecular free volume, respectively. In Eq. (1) the molecular free volume has been expressed within the quasi-harmonic approximation in terms of the lattice and the potential parameters [16].

Potential models for the crystalline phases of polymeric nitrogen having A7 or CG structure always assume that since the transition of a molecular phase into the polymeric phase is accompanied by breaking of the triple bond and forming of three single bonds, each atom has the first coordination sphere formed by exactly three neighbors. In the case of A7 lattice it is a layered structure, and in the case of CG - a spatial polymeric structure.

In the first case, the model expression for the interaction energy has the form [16]

$$
U_{N}=\frac{1}{2} \sum \sum_{1 \leq i<j \leq N} \Phi\left(r_{i j}\right)+\sum_{\text {bonded }} U_{1}\left(L_{i j}\right) .
$$

The first summation here is taken over all pairs of atoms, the second one - only over those who are nearest neighbors and are bound by single bonds ( $L_{i j}$ are the instant lengths of these bonds). As the origin of the energy here the energy of isolated atoms are used. 
To calculate thermodynamic properties of the polymeric nitrogen crystalline phase, having the structure A7, a canonical equation of the classical crystalline was used [16]. The expression for the free energy in the first-order theory has a form similar to the above equation molecular phase:

$F^{(\mathrm{at})}=F_{\mathrm{at}}^{(\mathrm{id})}+U_{\text {rep }}+\Delta U_{1}\left(L_{0}\right)-N_{a} k T \ln \left(\frac{v_{f}^{(\text {at })}}{v_{1}}\right)$.

Here $F_{\text {at }}^{(\text {id) }}(T, V)$ is the energy of an ideal atomic lattice gas as a function of temperature and volume, $\Delta U_{1}\left(L_{0}\right)$ is the energy of the A7 static lattice, $L_{0}$ is the equilibrium length of a (single) chemical bond, $v_{1}=V / N_{a}$ and $v_{f}{ }^{\text {(at) }}$ are the specific and atomic free volume, respectively. The quantities $\Delta U_{1}\left(L_{0}\right)$ and $v_{f}{ }^{\text {(at) }}$ in Eq. (3) were expressed in terms of the lattice parameters and parameters of the interaction potentials in Eq. (2).

Using the explicit expression for the free energy Eq. (3) and conventional thermodynamic relations, one can calculate all thermodynamic functions, including chemical potential and pressure necessary for the calculation of the phase equilibria.

A slightly different approach was used to develop the potential model for the polymeric CG crystalline structure of the nitrogen observed in experiments. To describe the nonbonded interactions of all pairs of nonbound atoms in the crystal the same atom-atom force model as applied to polymeric A7 structure was used. But the valence forces acting between bound atoms were described more detailed by a function depending both on the bond lengths and bond angles between pairs of single bonds attached to atoms. Parameters of such model were calibrated to reproduce the results of testing the sensitivity of the CG crystal energy, obtained in $a b$ initio quantum mechanical calculations [5], to the deformation of bonds and variation of bond angles. This potential model was used in Monte Carlo computer simulations [19] of polymeric CG solid (512 atoms in the box and periodic boundary conditions). The simulation results, in turn, were used in [18] to determine the parameters of the equation of state for CG phase.

The results of Monte Carlo simulations [19] indicate that the polymeric CG structure exhibits at high densities the negative values of thermal expansion coefficient. This specific behavior of thermodynamic properties cannot be reproduced by the canonical equation similar to Eq. (3).

To describe the specific behavior of thermodynamic properties, in particular the negative thermal expansion, in Ref. 18 was suggested a new canonical equation of state for CG polymer phase of solid nitrogen. Helmholtz free energy was represented as a sum of two contributions, quasi-harmonic and anharmonic ones:

$$
F^{(\text {at })}=F^{(h)}+\Delta F^{(\text {anh })} .
$$

The main contribution to the Helmholtz free energy in Eq. (4) is the quasi-harmonic contribution $F^{(h)}$ described by the modified Mie-Grüeneisen model:

$$
F^{(h)}=U_{1}^{(C G)}+3 N_{a} k T D_{F}\left(x_{D}\right)
$$

Here $U_{1}^{(C G)}$ is the energy of the static lattice, $x_{D}=\Theta_{D} / T$, $D_{F}\left(x_{D}\right)$ is Debye function, and $\Theta_{D}$ is the Debye temperature. The Grüeneisen parameter $\gamma=d \ln \Theta_{D} / d \ln \rho$ was specified as a linear function of density: $\gamma=\gamma_{0}\left(1-\rho / \rho_{0}\right)$. This model contains two constants: $\gamma_{0}$ and $\rho_{0}$. These constants were determined using the above-mentioned Monte Carlo data [19].

Anharmonic effects in the solid polymeric nitrogen at high temperatures may be very significant [19]. The contribution of anharmonic effects to the free energy $\Delta F^{(a n h)}$ was determined by the deviations of the specific heat from the Dulong-Petit law, found in computer simulations [20].

\section{Phase equilibria of the polymeric and molecular phases in solid nitrogen}

Equations of state for both molecular and polymeric phases were used in Ref. 18 to determine $T(P)$-dependence of the polymerization phase transitions. In the calculation of the parameters of the phase transition, the conditions of material equilibrium, i.e., the equality of the chemical potentials (per atom) and mechanical equilibrium (equality of pressure for both phases) were used. Because coexisting phases were described by two separate equations of state based on different potential models they must have a common origin of energy. Therefore, the chemical potential of the polymeric phase was calculated by:

$$
\mu^{(\mathrm{at})}(T, V)=\frac{\partial F^{(\mathrm{at})}(T, V)}{\partial N_{\mathrm{at}}}+\Delta \mu_{0} .
$$

Here $\Delta \mu_{0}=-0.98 \mathrm{eV} /$ atom is the difference in the origins of the polymeric and molecular phases [5].

Calculation of the phase equilibrium was carried out numerically using the standard method of double tangent, i.e., the equilibrium pressure of phase transition at a given temperature $P(T)$ was determined by the slope of the common tangent for to $F_{\text {mol }}(T, V)$, and $F^{\text {(at) }}(T, V)+\Delta \mu_{0}$ curves versus volume and orthobaric volumes - by abscissae of these tangency points.

Results of such calculations are illustrated in Fig. 1, where two $T(P)$-curves for two types of polymeric structures - CG and A7 - are presented. As can be seen, the equilibrium pressure of the transition into the $\mathrm{A} 7$ structure increases monotonically with increasing temperature, and $P(T)$-dependence in the case of CG structure is just the opposite. As it was shown in Ref. 18, these differences are explained by the negative thermal expansion found in CG phase.

In the case of the $C G$ polymeric structure the phase transition line $T(P)$ intersects with the melting line at 


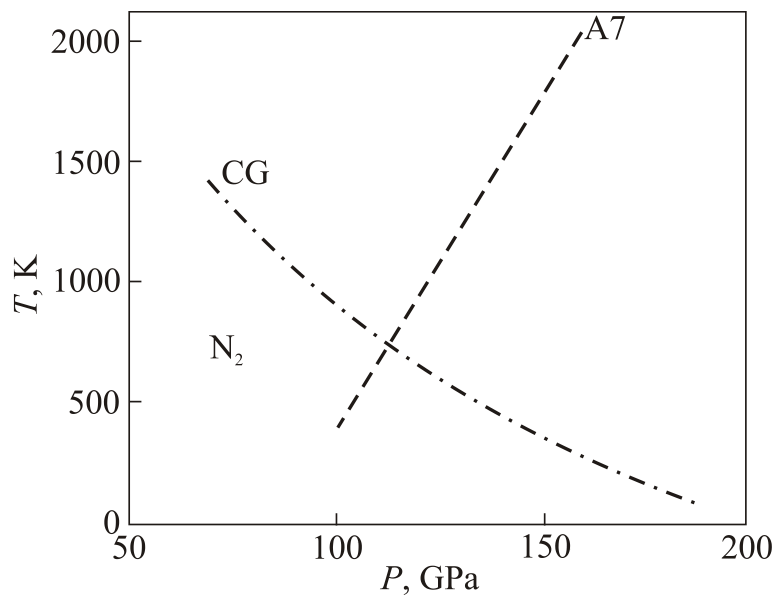

Fig. 1. Calculated dependences of the equilibrium phase transition temperature of solid nitrogen from the molecular phase into polymeric phase as a functions of pressure. Dashed line corresponds to A7 and the dash-dotted line to CG structure.

$T \sim 1500 \mathrm{~K}$ and at a relatively low pressure of about $50 \mathrm{GPa}$. It is interesting to note that the hypothetical triple point (solid molecular nitrogen-solid polymeric liquid nitrogen) predicted in Ref. 18 is approximately in the same region, where, according to measurements of Mukherjee and Boehler [23], as well as of Goncharov et al. [22] the maximum of melting temperature was observed.

According to Ref. 18, the latent heat of polymerization transition $L$ into CG phase increases approximately linearly with temperature, like the case of the A7 structure but has the opposite sign. This means that the entropy jump $\Delta S=L / T$ is almost constant. At the same time, unlike the $\mathrm{N}_{2}-\mathrm{A} 7$ transition, heat of transition is negative. At $T \sim 1000 \mathrm{~K}$ the latent heat reaches $L \sim-1.0 \mathrm{eV} / \mathrm{atom}$, when the predicted heat of transition in the structure A7 is about $0.7 \mathrm{eV} /$ atom. There is also a significant difference in the orthobaric volumes behavior of coexisting phases. In the case of $\mathrm{N}_{2}-\mathrm{A} 7$ transition these volumes slightly decrease with temperature. In the case of transition into the polymeric CG phase both orthobaric volumes $V_{\text {mol }}$, and $V_{\text {poly }}$, and the jump in volume $V_{\text {mol }}-V_{\text {poly }}$ increase with temperature. Thus, in evaluating the thermodynamic behavior of the molecular-to-polymeric crystal transition at nonzero temperature the type of the structure of polymeric phase is very important.

In Fig. 2 the calculated orthobaric volumes [18] are compared with the experimental data [21] at room temperature. This comparison shows that the calculated volumes of both molecular and polymeric phases, although somewhat overestimated, are in reasonable agreement with existing measurements of Eremets et al. [9,10,21], obtained in the diamond anvil cell at room temperature and pressures up to 2 Mbar.

The predicted value of the volume jump is very close to the measured. Eremets et al. found 22\% of volume change, theoretical result was about $21 \%$. Predicted volume of the po-

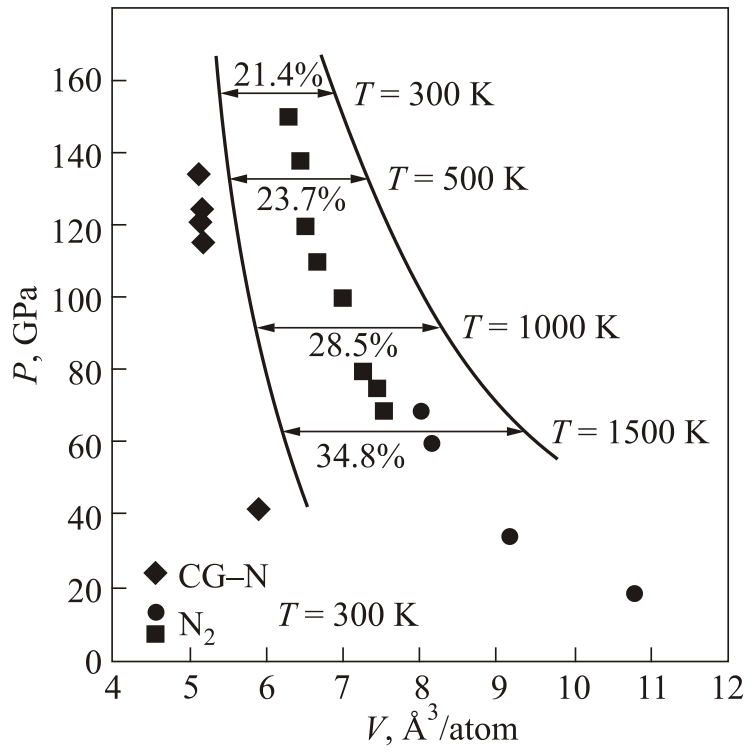

Fig. 2. Comparison of the calculated orthobaric volumes of the coexisting molecular and CG polymeric phases [18] with the available experimental data at room temperature [21].

lymeric phase agrees with the experimental value at room temperature within $2 \%$ and the volume of the molecular phase within $6 \%$.

\section{Evaluation of the melting line of polymeric nitrogen}

The generality of the idea, which explains the phenomenon of polymerization in the liquid and solid phases, allows using canonical equations of state of two phases, to predict the transition line where the crystalline polymeric nitrogen melts forming polymeric liquid. Such a prediction was suggested in Ref. 25 where two canonical equations of state: the modified equation of Mie-Grüeneisen Eq. (5) for the solid polymeric CG nitrogen, and the equation of state for the liquid polymeric nitrogen proposed in Ref. 26 were used.

The parameters of the last equation of state were determined by reproducing the results of the recent $a b$ initio simulations of Boats and Bonev [20], including the predicted location of the liquid-liquid coexistence line in highly compressed nitrogen.

The expression for the Helmholtz free energy of the polymerizing liquid generalizes the canonical equation of state for polymeric nitrogen fluid, proposed earlier in Ref. 14. The expression for the Helmholtz free energy has the following form:

$$
F=F_{\text {poly }}^{(\mathrm{id})}(\alpha)+\Delta F^{(H D)}\left(\eta_{1}\right)+\alpha \Delta F_{\text {poly }}\left(\eta_{L}\right) .
$$

Here $F_{\text {poly }}^{(i d)}(\alpha)$ is the free energy of an ideal mixture of monomers (N2), dimers N4, etc., an explicit expression for which was obtained in Ref. 26, and $\alpha$ is the degree of polymerization, (i.e., portion of all molecules bound in all types of clusters). Functions $\Delta F^{(H D)}\left(\eta_{1}\right)$ and $\Delta F_{\text {poly }}\left(\eta_{L}\right)$ express the correction on nonideality of the mixture of hard 
dumbbells and the excess free energy of polymeric fluid [14], correspondingly. Respectively, they are dependent on two types of packing fractions: "molecular" $\eta_{1}=\pi n d^{3} / 3$ and "polymeric" $\eta_{L}=\pi n d_{L}^{3} / 3$ ones, where $d$ is the effective molecular diameter and $d_{L}$ is the effective diameter of an atom in the polymeric liquid. Parameters of this equation of state have been determined in Ref. 26 and reproduce the results of $a b$ initio simulations [20]. In particular, the liquid-liquid separation line in partially polymerized nitrogen liquid with critical point at $T_{C}=4890 \mathrm{~K}$ [26].

Equations of state Eqs. (7) and (3) were used in [25] to find pressure dependence of the melting temperature $T_{m}$ of CG crystalline polymer, orthobaric volume of solid and liquid phases, and latent heat of fusion. Results of calculating $T_{m}(P)$ are presented in the phase diagram (Fig. 3) in comparison with existing experimental data on melting line of nitrogen $[23,24]$. As one can see in Fig. 3, near to $T=1700 \mathrm{~K}$ and $P=70 \mathrm{GPa}$ a triple point of the crystal polymer-polymer liquid-molecular liquid-type is formed.

As it was already noted above, the equation of state for the liquid polymeric nitrogen Eq. (7) was calibrated on $a b$ initio simulation data presented by Boats and Bonev [20] in a temperature range 2000-5000 K. Therefore, the calculations in Ref. 25 were performed only within a reasonable limited extrapolation to $T<2000 \mathrm{~K}$ and hence cover a limited range of temperatures and pressures.

Despite this limitations, an important qualitative feature of the predicted $T_{m}(P)$ dependence should be noted - it decreases with increasing pressure, like the transition temperature of molecular crystal into the polymeric CG phase. Predicted melting temperature falls from $1750 \mathrm{~K}$ at $80 \mathrm{GPa}$ up to $1500 \mathrm{~K}$ at $95 \mathrm{GPa}$. This behavior is in agreement

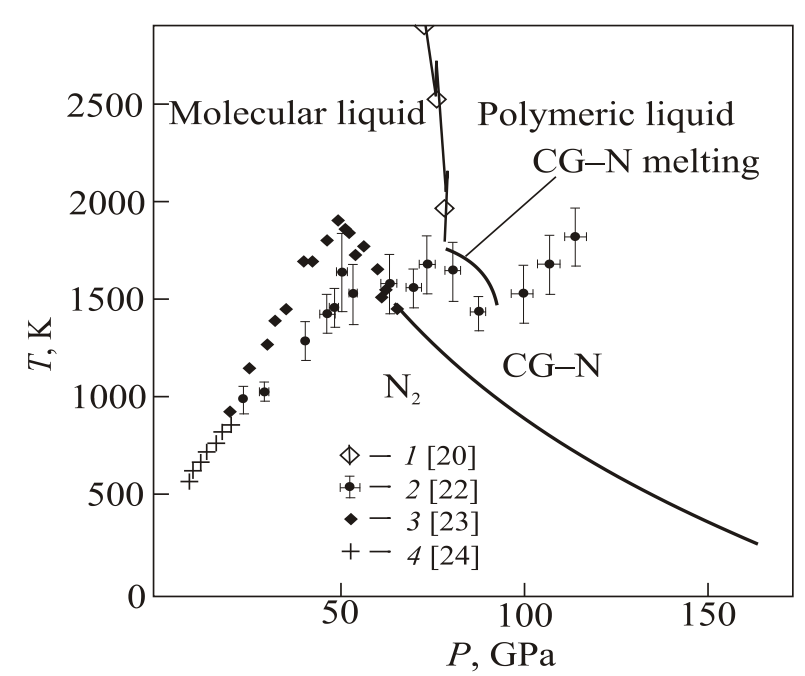

Fig. 3. Phase diagram of strongly compressed nitrogen. The solid lines show the calculated transition line from the molecular to CG polymeric phase, the melting line of the polymeric solid and the transition line liquid-liquid in partially polymerized nitrogen: 1 are the computer simulation results [20], 2-4 are the experimental data on the melting line. with the recent measurements of Goncharov et al. [22], who observed a maximum melting temperature in this pressure range. The predicted value of the latent heat of the CG crystal melting is also negative and its absolute value increases with increasing temperature. At $T=1700 \mathrm{~K}$, the value of the latent heat reaches $-2.0 \mathrm{eV} / \mathrm{atom}$ [25].

\section{Discussion and conclusions}

Phase transitions of simple highly compressed homonuclear systems from the molecular into the nonmolecular forms (dissociation, ionization, polymerization) have been long attracted the attention of scientists as a promising way for obtaining materials with high density of energy. Study the conditions of formation of such high-energy-density phases requires, inter alia, studying phase diagrams of such systems at extreme pressures, where such transitions may occur. In the recent decades, the main attention of experimentalists in this direction was attracted to investigating the possibility of polymerization of molecular nitrogen in the crystalline state. However, the objective difficulties of such research at ultrahigh pressures do not allow complete solving of this problem using experimental methods only.

Theoretical study of these transitions and prediction of their location in the phase diagram at high pressures and temperatures is a problem which can be solved by invoking canonical equations of state based on the ground of statistical physics. Successful development of such equations of state depends on the underlying microscopic theoretical models. Practical application of the theory is always strongly dependent on the adequacy of potential models, used for description of atomic and molecular interactions in a solid, and on the possibility of their calibration on reliable $a b$ initio quantum mechanical calculations.

A number of potential models, focused on the description of the specificity of the interaction in the studied systems have been applied to predict the positions of lines of phase transitions accompanied by dissociation of nitrogen molecules and formation of a spatial network of covalent bonds between atoms.

Such potential models, calibrated on the limited $a b$ initio calculations of the static lattice energy of systems studied, which correspond to their state at zero temperature, can be successfully applied in conventional computer simulations (e.g. Monte Carlo or molecular dynamics), which are able to predict the behavior of high-energy-density systems at elevated temperatures. An example of a successful application of this approach is the Monte Carlo simulation of the spatially-polymerized CG nitrogen [20], where the effect of the negative thermal expansion in the crystalline and amorphous structures was discovered.

As it was revealed later [25], this fact plays an important role not only in explaining the specific features of the thermodynamic behavior in polymeric phase, but also in configuration of the phase diagram of nitrogen at high pressures. 
Pressure dependence of the transition temperature into the spatial-polymerized high-pressure CG phase of nitrogen, having negative thermal expansion at high densities, has a negative slope, while the same dependence of the phase transition into the (hypothetical) layered polymeric A7 structure will be monotonically increasing, if such phase were stable.

It should also be noted that the effects of negative thermal expansion is manifested also in the negative slope the predicted pressure dependence of the melting temperature of the polymeric CG nitrogen shown in Fig. 3. Although in some aspects the parameters of this phase diagram differ from the predictions of some computer $a b$ initio calculations, in general, as shown above, they are in reasonable agreement with the available limited experimental data [21,22].

1. J.A. Ciezak, Metastable Polymeric Nitrogen: The Ultimate Green High-Energy-Density Material, ARL-TR-4478 Report, June 2008.

2. H.B. Radousky, W.J. Nellis, M. Ross, D.C. Hamilton, and A.C. Mitchell, Phys. Rev. Lett. 57, 2419 (1986).

3. M.K. McMahan and R. Le Sar, Phys. Rev. Lett. 54, 1929 (1985).

4. R.M. Martin and R.J. Needs. Phys. Rev. B 34, 5082 (1986).

5. C. Mailhiot, L.H. Yang, and M.K. McMahan, Phys. Rev. B 46, 14419 (1992).

6. A.F. Goncharov, E. Gregoryanz, H.-K. Mao, Z. Liu, and R. Hemley, Phys. Rev. Lett. 85, 1262 (2000).

7. M.I. Eremets, R.J. Hemley, H-K. Mao, and E. Gregoryanz, Nature 411, 170 (2001).

8. E. Gregoryanz, A.F. Goncharov, R.J. Hemley, H-K. Mao, M. Somayazulu, and G. Shen, Phys. Rev. B 66, 224108 (2002).
9. M.I. Eremets, A.G. Gavriliuk, I.A. Trojan, D.A. Dzivenko, and R. Boehler, Nature Materials. 3, 558 (2004).

10. M.I. Eremets, A.G. Gavriliuk, and I.A. Trojan, Appl. Phys. Lett. 90, 171904 (2007).

11. Physics of Cryocrystals, V.G. Manzhelii and Yu.A. Freiman (eds.), American Institute of Physics: Woodbury, NY (1997).

12. J. Hooper, A. Hu, F. Zhang, and T.K. Woo, Phys. Rev. B 80, 104117 (2009).

13. X. Wang, F. Tian, L. Wang, T. Cui, B. Liu, and G. Zou, J. Chem. Phys. 132, 024502 (2010).

14. E.S. Yakub, Zh. Fiz. Khim. 67, 305 (1993).

15. M. Ross and F. Rogers, Phys. Rev. B 74, 024103 (2006).

16. L.N. Yakub, Fiz. Nizk. Temp. 19, 531 (1993) [Low Temp. Phys. 19, 377 (1993)].

17. L.N. Yakub . J. Low Temp. Phys. 139, 783 (2005).

18. L.N. Yakub. Fiz. Nizk. Temp. 37, 543 (2011) [Low Temp. Phys. 37, 431 (2011)].

19. L.N. Yakub, J. Low Temp. Phys. 122, 501 (2001).

20. B. Boates and S.A. Bonev, Phys. Rev. Lett. 102, 015701 (2009).

21. M.I. Eremets, A.G. Gavriliuk, N.R. Serebryanaya, I.A. Trojan, D.A. Dzivenko, R. Boehler, H.-K. Mao, and R.J. Hemley. J. Chem. Phys. 121, 11296 (2004).

22. A.F. Goncharov, J.C. Crowhurst, V.V. Struzhkin, and R.J. Hemley. Phys. Rev. Lett. 101, 095502 (2008).

23. G.D. Mukherjee and R. Boehler. Phys. Rev. Lett. 99, 225701 (2007).

24. S. Zinn, D. Schiferl, and M.F. Nicol, J. Chem. Phys. 87, 1267 (1987).

25. L.N. Yakub, Fiz. Nizk. Temp. 39, 552 (2013) [Low Temp. Phys. 39, 427 (2013)].

26. E.S. Yakub and L.N. Yakub, Fluid Phase Equilibria 351, 43 (2013). 